
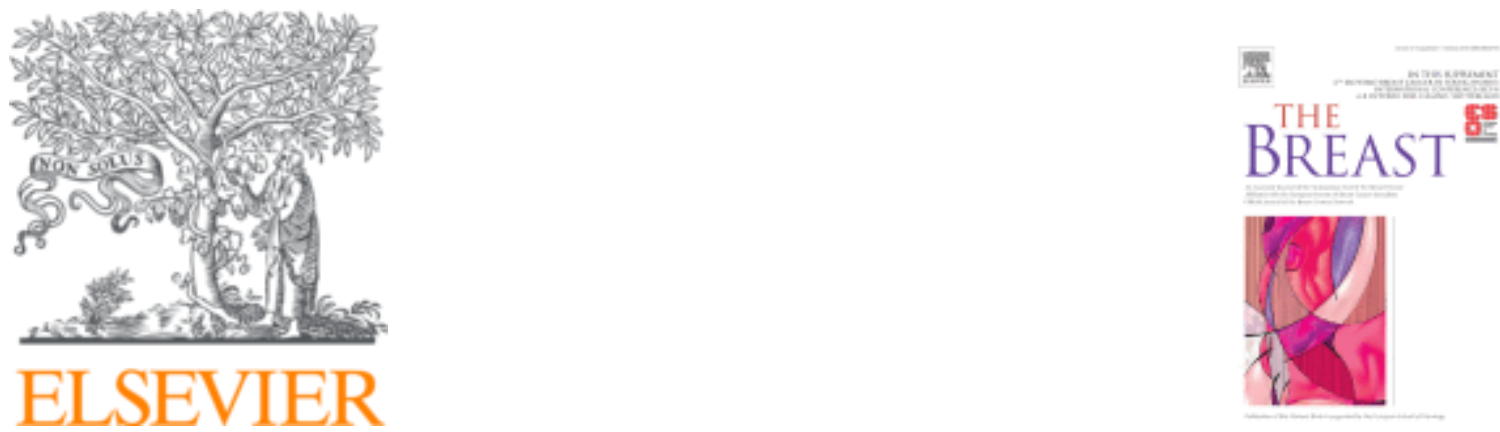

\title{
Can breast cancer metastasize to an unusual organ? : case report and review
}

Author links open overlay panel : MarijaKarakolevska - Ilova

Institute of Oncology and Radioterapy, Dept. of Medical Oncology, Stip, Macedonia

Available online 5 October 2018.

https://doi.org/10.1016/j.breast.2018.08.063

Copyright (C) 2018 Published by Elsevier Ltd.

Part of special issue:

4th ESO-ESMO Breast Cancer in Young Women International Conference (BCY4), 6 - 8 October 2018, Lugano, Switzerland: Abstract book

Copyright (C) 2020 Elsevier B.V. or its licensors or contributors. ScienceDirect ${ }^{\circledR}$ is a registered trademark of Elsevier B.V.

ScienceDirect ${ }^{\circledR}$ is a registered trademark of Elsevier B.V.

Introduction: Metastatic cancer in the thyroid is uncommon and large autopsy studies found out that the incidence of thyroid metastases in patients with a history of cancer ranges from $1.9 \%$ to $24 \%$, so in any patient with a previous history of malignancy, a new thyroid mass should be considered as recurrence until proved otherwise. The most frequent primary cancers are renal cell (48.1\%), colorectal (10.4\%), lung (8.3\%) and breast (7.8\%) cancers.Most patients with thyroid metastases have widespread metastatic disease but occasionally the thyroid may be the only site of disease with the same impact on prognosis as nonthyroidal metastases. Thyroid metastases from breast cancer can appear (7,8\%), but most of them are clinically irrelevant or occult.

Case presentation: This is the case of a 38 years old woman, premenopausa. In August 2015 radical mastectomy with dissection of the left axilla was performed (from 7 removed lymph nodes, 3 were positive with primer tumor size of $4 \mathrm{sm})$, pT2pN1Mx, Stage IIB.IHH:ER(40\%), PgR(50\%), Ki67 (25\%), p53 (5\%), Her2/neu (0\%). CT with i.v contrast of the thorax and abdomen were normal. In Sept. 2015 the patient was set on chemotherapy 
with $4 \mathrm{AC}+4 \mathrm{P}$, after that RT $(50 \mathrm{~Gy} / 25 \mathrm{fr})$ was planned. The patient refused the RT and she was set on the hormonal therapy with LHRH agonist and Tamoxifen 10mg 2x1 (april 2016).The patient suffered for the severe pain in her chest, spine and legs (may 2016). MRI of the spine showed degenerative changes at Th11-12 and CT of lung and abdomen were also normal. The pain in the bones got worse, so bone scan with Tc (August 2016) was performed: no pathological accumulation. 11 months after the hormone therapy was started, floating spherical formation appeared under operating scar (fine-needle biopsy -classif. group 5). CT of the lung and abdomen were normal. The patient was operated and the result was carcinoma mammae metast.lymphogland.axillae.IHH:ER(60\%),PgR (0\%),Ki67(35\%), Her2/neu(0\%). Chemotherapy with CMF protocol was started and after that RT(50Gy/25fr). At the first follow-up, clinical examination showed enlarged thyroid lobes with normal function. Ultrasound revealed a large nodule in left lobe with mixed echo-pattern. The cytology revealed malignant cells, left thyroidectomy was done and ms were confirmed(HER2+).

\section{Discusion:}

Reviewing the literature, only 43 cases of breast cancer with thyroid metastases are reported thus far. While clinical and histopathological features of TM were well described in the literature, radiological publications consist predominantly of small series. There are no case reports that describe the type of systemic treatment used. Surgical resection of an isolated metastasis may result in prolonged disease-free survival. Every thyroid node in cancer patient should be consider as malignant.

\section{References:}

1.Harris A, Taylor O. Agbaje H, GarmocIncidence of bone metastases and survival after a diagnosis of bone metastases in breast cancer patients. Cancer Epidemiology.Volume 38, Issue 4, Aug 2014:pages 427-434

2.Clayton AJ, Danson S, Jolly S, Ryder W, Burt P, Stewart A, et all. Incidence of cerebral metastases in patients treated with trastuzumab for metastatic breast cancer. British Journal of Cancer. Volume 91, July 2004:pages 639-643.

3.Nakhjavani MK, Gharib H, Goellner JR, van Heerden JA: Metastasis to the thyroid gland. A report of 43 cases. Cancer. Volume 79. 1997:pages 574-578.

4.Yang S,Park K, Kim J. Thyroid Metastasis from Breast Carcinoma Accompanied by Papillary Thyroid Carcinoma. Case Rep Oncol. Volume 7. 2014:pages 528-533

5.Mei Y. Wei W.Chenfang Z. Thyroid gland metastasis arising from breast cancer: A case report. Oncology Letters.2013: pages 1836-1838. 